\title{
Auditory detection and recognition in a two-alternative, directional uncertainty situation
}

\author{
G. LOWE 1 \\ UNIVERSITY OF HULL, England
}

The effects of directional uncertainty on the detection and recognition of auditory signals were investigated, with particular reference to predictions from the Luce-Shipley choice theory analysis. Sixteen Ss were used in a group detection and recognition task, involving auditory signals in a white noise background, from two alternative sources to the right and left of the Ss. Confidence ratings and false alarm rates were used to calculate detectability measures, and from confusion matrices signal recognition and response bias parameters were estimated. The results indicated that detectability is reduced by uncertainty of this kind, provided the initial detectability of the signal is fairly high. However, recognition was apparently unrelated to detection performance, except in very general terms. Certainly the data revealed no evidence for a precise, quantifiable relation.

Existing data on the detection of auditory signals indicate that uncertainty with respect to the parameters of the signal will result in a significant decrement in the detectability of that signal (e.g., Creelman, 1960; Veniar, 1958; Swets \& Birdsall, 1956). An experiment by Gundy (1961) on the auditory detection of unspecified signals showed that performance was at chance level initially, but showed gradual improvement as the experiment progressed. Whereas when the signal was specified by presenting it $10 \mathrm{~dB}$ louder than threshold level before each test sequence, Ss maintained a stable level of performance. This could be interpreted as a kind of "cueing" effect, which Shipley (1959) has studied. She suggests that a given trial can offer cues to the nature of the signal. The adequacy of the cue is presumably determined by the effective signal intensity.

Temporal uncertainty of an auditory signal also results in a decrease in detectability (Egan, Greenberg, \& Schulman, 1961). However, much of the work on signal uncertainty has been concerned with frequency uncertainty, and this has given rise to several alternative models of frequency detection. The "singleband" model (Tanner et al, 1956) suggests that the observer acts as a "narrow band-pass filter or receiver," monitoring a single, limited range of frequencies at any given time. On the other hand, the "multi-band" model (Green, 1958) makes the additional assumption that more than one of these narrow filters can be utilized at any one time, i.e., simultaneous monitoring of several different frequency ranges. These models are, of course, specifically designed to deal with frequency detection, but the notion of monitoring a single or multiple input may be applied to other (auditory) detection tasks, where "tuning" or "cueing" is possible; for instance, in the case of directional uncertainty. Models of this kind lead to differential predictions regarding the decrement in detectability resulting from signal uncertainty, particularly in relation to the false alarm (FA) rate. In a detection situation with two alternative (Left or Right) directional uncertainty of the signal, the observer's FA rate should increase if he is "tuned in" to both signal sources. On the other hand, with monitoring restricted to one single source at a time, the observer would be "tuned in" to the wrong signal source on some trials, thereby increasing the number of "missed" signals. The existing data on frequency uncertainty do not seem to support either of these models unequivocally, but there is very little information on the extension of such models to a directional uncertainty situation.

Shipley (1960) offers an alternative explanation of the decrement in detectability due to signal uncertainty. It is basically an extension of Luce's Choice model (1959), and applies to any signal detection situation, whenever detection and recognition are both possible. She assumes that even a simple detection task involves implicit recognition responses. It is argued that, if one takes the full stimulus-response (S-R) confusion matrix based on recognition responses (implicit or otherwise), and collapses this into a simple detection matrix, the result will be a reduction in the proportion of correct detections; provided, however, the confusion parameters of the signals are less than 1, i.e., provided there is a significant degree of discriminability between the alternative signals. This model is based upon an analysis of response determinants and may be easily generalized to detection and recognition situations involving any type of signal uncertainty, in contrast to the "filter" models of Green and Tanner, which are specifically related to frequency uncertainty.

The main aims of the present experiment were as follows:

(1) to investigate the effect of directional uncertainty on the detection and recognition of auditory signals. The uncertainty consisted of two alternative signal sources, either to the right or left of the Ss.

(2) to examine predictions made by the Luce-Shipley Choice model; (a) that the detectability of a given signal may be reduced by signal uncertainty, whatever the nature of this uncertainty; (b) that the size of this decrement is related to the relative discriminability of the alternative signals.

(3) if such a decrement is found, it would suggest some sort of "perceptual tuning." In this case, examination of the observed FA rates may give some indication as to whether this took the form of a single input or simultaneous dual input monitoring system.

(4) to investigate, and, if possible, quantify the way in which detectability is related to the confusion and recognition parameters of the stimulus situation.

\section{METHOD}

The experiment involved a detection and recognition task, using a $750 \mathrm{msec}, 1000 \mathrm{~Hz}$ tone, from one of two loudspeakers situated to the right and left of the group of Ss, who were centrally placed in a large room. White noise was generated through other loudspeakers to the front and rear of the Ss. Signal presentation time and duration were indicated by a red warning light at the front of the room. There were two signal intensities; Intensity 1 being $4 \mathrm{~dB}$ louder than Intensity 2 . These levels were determined in a preliminary session of 100 trials, and corresponded, approximately, to $75 \%$ and $50 \%$ detection thresholds. Signal intensities and blank trials (B) were randomized, and stimulus presentation was controlled by means of a programmed input to a tape-reader.

There were three conditions: (1) signal specified Left (SS:L); (2) signal specified Right (SS:R); and (3) signal uncertain (UNC), Left or Right. These conditions were presented twice in a balanced design with 8 blocks of 40 trials. There were 40 trials for each signal in each condition, and in addition there were as many blank trials [i.e., $p(S)=p(B)=0.5$ ]

Sixteen undergraduate students, unpaid and relatively inexperienced in this sort of detection task, acted as Ss. They were required to use a 4-point rating scale, ranging from ' $O$ ' =

\section{Table 1}

Average d' values and average False Alarm rates for 16 Ss under conditions of uncertainty (UNC) and no uncertainty (SS).

\begin{tabular}{cccccc}
\multicolumn{5}{c}{ Average d'values } \\
SS: & L1 & L2 & R1 & R2 & Av. \\
UNC: & 1.25 & 0.85 & 0.26 & 0.27 & 0.66 \\
& 1.16 & 0.70 & 0.43 & 0.06 & 0.59 \\
& \multicolumn{5}{c}{ False Alarm Rate: $\mathbf{P}(\mathbf{Y} / \mathbf{B})$} \\
& SS: $\mathbf{R}$ & \multicolumn{2}{c}{ SS:L } & UNC & \\
& .141 & .120 & .115 & \\
\hline
\end{tabular}


Table 2

Overalt S-R confusion matrix for 16 Ss based on recognition responses in the uncertainty (UNC) condition. Estimates of $d^{*}$ and $\mathrm{Cl}$ are derived directly from the matrix.

\begin{tabular}{crcrr}
\multicolumn{5}{c}{ Response } \\
Stimulus & L & R & N & \\
LS & .38 & .28 & .34 & $\mathrm{~d}^{\prime}=0.57$ \\
RS & .15 & .33 & .52 & $\mathrm{Cl}=0.58$ \\
B & .11. & .13 & .76 &
\end{tabular}

"definitely no signal" to ' 3 ' = "definitely a signal present." In addition, recognition responses ( $L$ or $R$ ) were required in the $U N C$ condition, for all trials, even when the $\mathrm{S}$ gave a "no detection" response ('0').

\section{ANALYSIS OF DATA}

For each $S$, measures of detectability, $d^{\prime}$, were obtained from the ratings in the SS and UNC conditions, with FA rates directly observable (Table 1). For the UNC condition, the data were separated into two classes of S-R confusion matrix: (1) a matrix for undetected signals (i.e., for those signals which the $\mathrm{Ss}$ rated ' 0 ', but because of the instructions, were forced to make a $L / R$ "recognition" response. These constituted about $40 \%$ of all signals presented in the UNC condition (Table 4); and (2) a matrix for all trials, with all ratings of ' 0 ' classified as "no detection" $(N)$, ignoring the forced $L / R$ recognition response (Table 2). Furthermore, by collapsing this second matrix (treating LS and RS as $S$, and $L$ and $R$ responses as a simple detection response, $Y$ ), an additional measure of signal detectability in the UNC condition could be obtained (Table 2). Separate confusion matrices were also calculated for each signal intensity (Table 3 ).

Indices of signal confusion (CI), inversely related to the discriminability or relative recognizability of the signals, were calculated from both individual and combined (average) S-R confusion matrices,

$$
\text { where } C I=\frac{\frac{p(R / L)}{p(R / L)+p(L / L)}+\frac{p(L / R)}{p(L / R)+p(R / R)}}{\frac{p(L / L)}{p(L / L)+p(R / L)}+\frac{p(R / R)}{p(R / R)+p(L / R)}}
$$

Finally, a measure of response bias could also be obtained from the confusion matrices, i.e., $\mathrm{p}(\mathrm{R} / \mathrm{B}): \mathrm{p}(\mathrm{L} / \mathrm{B})$, in Table 2 .

\section{RESULTS AND DISCUSSION}

The overall, average detectability for all Ss for all classes of signal was slightly lower in the UNC condition, but this decrement was not significant (see Table 1). However, these values were based on the average detectabilities of the four types of signal, taking the group of $16 \mathrm{Ss}$ as a whole. But, since there were $16 \mathrm{Ss}$, each occupying a different position with respect to the signal sources ( $L$ or $\mathrm{R}$ speakers) and white noise sources, there was, in effect a variety of different $S / N$ ratios, resulting in a considerable range of detectability values. This enabled one to make a more detailed analysis in terms of whether more detectable and less detectable signals were differentially affected by uncertainty.

Detectability values for each $\mathrm{S}$ were divided into two groups according to whether they were above or below the average $\mathrm{d}^{\prime}$ value for that particular signal in the SS conditton. These two groups were then analyzed in terms of a decrement value obtained from the difference between $d^{\prime} S S$ and $d^{\prime} U N C$ (Fig. 1). It can be seen that those signals which did show a significant decrement

\section{Table 4}

Conditional probability matrix for undetected signals (given a rating ' $O$ ' but with forced recognition responses ). Overall average for $16 \mathrm{Ss}$.

\begin{tabular}{ccc} 
& \multicolumn{2}{c}{ Response } \\
Stimulus & L & R \\
LS & .413 & .587 \\
RS & .325 & .675 \\
\hline
\end{tabular}

were more detectable in the SS condition: less detectable signals showed no decrement. This result would be in line with Shipley's hypothesis, as long as one assumes that signal detectability is directly related to signal discriminability or recognition.

The next step, therefore, was to analyze the recognition data from the UNC condition, in terms of a S-R confusion matrix, and determine whether recognition or confusion parameters were, in fact, related to the detectability of the signals. Table 2 indicates that signal recognition was only moderate, with an overall average confusion index of 0.58 . However, one can break this recognition data down further in terms of the two signal intensities. Table 3 shows the separate confusion matrices, together with confusion indices and detectability measures derived from the collapsed matrices. From this it appears that there is some indication of a relationship between detectability and confusion parameters, since the greater the detectability of the signals, the more recognizable they are.

However, this only gives a very rough indication, since the data from all $16 \mathrm{Ss}$ were combined and averaged in these matrices. Therefore, in order to examine this apparent relationship more precisely, an analysis was made of the confusion matrices of individual Ss. Individual confusion indices were obtained and related to detectability measures derived from both ratings and collapsed S-R matrices (Fig. 2). It seems evident that signal confusion/recognition parameters are not specifically related to either measure of detectability, Even if one takes a cruder measure

\section{Table 3}

S-R confusion matrices with derived $d^{\prime}$ and $C I$ values for signal intensities 1 and 2 (average for $16 \mathrm{Ss}$ ).

\begin{tabular}{|c|c|c|c|c|}
\hline \multicolumn{5}{|c|}{ Response } \\
\hline Stimulus & $\mathbf{L}$ & $\mathbf{R}$ & $\mathbf{N}$ & $\mathrm{p}(\mathrm{Y} / \mathrm{S})=.505$ \\
\hline LS & .34 & .28 & .38 & $\mathrm{p}(\mathrm{Y} / \mathrm{B})=.24$ \\
\hline RS & .12 & .27 & .61 & $\mathrm{~d}^{\prime}=.72$ \\
\hline \multirow[t]{3}{*}{ B } & .11 & .13 & .76 & $\mathrm{CI}=.60$ \\
\hline & \multicolumn{4}{|c|}{ Signal Intensity 2} \\
\hline & $\mathbf{L}$ & $\mathbf{R}$ & $\mathbf{N}$ & $\mathrm{p}(\mathrm{Y} / \mathrm{S})=.65$ \\
\hline LS & .44 & .30 & .26 & $\mathrm{p}(\mathrm{Y} / \mathrm{B})=.24$ \\
\hline RS & .16 & .41 & .43 & $d^{\prime}=1.09$ \\
\hline \multirow[t]{2}{*}{ B } & .11 & .13 & .76 & $\mathrm{CI}=.52$ \\
\hline & \multicolumn{4}{|c|}{ Signal Intensity 1} \\
\hline
\end{tabular}

of detection-the 'hit' rate, $\mathrm{p}(\mathrm{Y} / \mathrm{S})$, this is also unrelated to the confusion/recognition parameter (Fig. 3).

Examination of the FA rates (Table 1) shows that the overall average FA rate did not increase in the UNC condition-indeed, there is a slight decrease. Closer analysis of individual FA rates indicates that, where decrements in detectability did occur, these were attributable, in general, to a combination of a slightly increased FA rate and a concomitant decrease in frequency of detection responses, $p(Y / S)$. Only two out of nine Ss showing decrements had increased FA rates as the sole determinant of the decrement: in two other Ss, the decrement was directly due to a decrease in 'hit' rate.

An increase in FA rate in the UNC condition would be evidence for a "dual input monitoring system," provided there was a significant decrement in detectability due to uncertainty. Since the overall analysis reveals no such decrement, the FA rate and 'missed signal' rate are thus inadequate factors in the distinction between mechanisms of single or dual input monitoring. In any case, signal duration is likely to be an important factor. For instance, Swets (1963) has shown, in relation to frequency uncertainty, that decrements in $\mathrm{d}^{\prime}$ are inversely related to signal duration. If one assumes that a similar relation exists for directional uncertainty, then it is not surprising that no significant decrement was observed in the present experiment, since signal duration was relatively long $(0.75 \mathrm{sec})$.

A final point with regard to Shipley's hypothesis is the assumption that there can be no recognition without detection. In other words, undetected signals should show no differential identification. Examination of responses to undetected signals reveals that the proportion of correct recognition responses was 


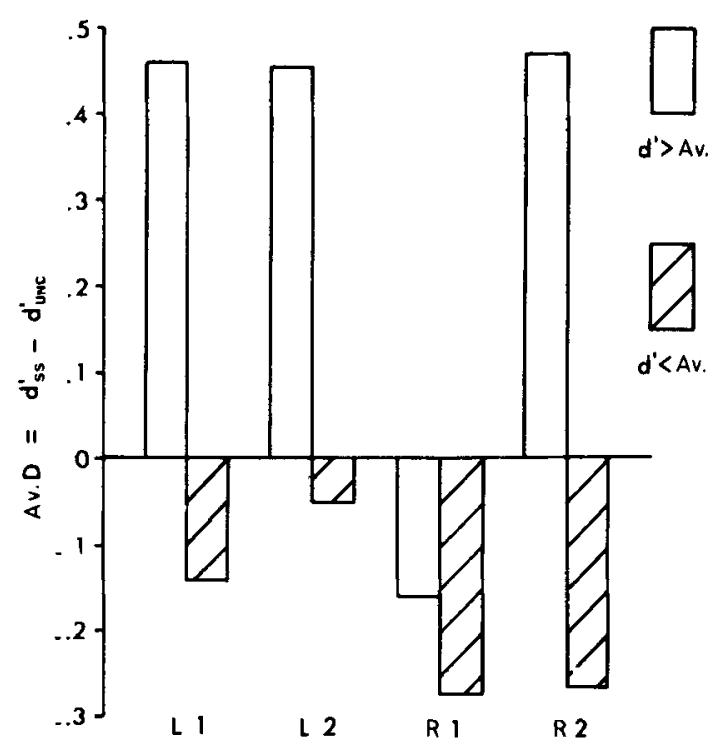

Fig. 1. Average detectability decrement as a function of relative signal detectability.

not significantly better than chance (Table 4).

In conclusion, then, it seems that directional uncertainty of auditory signals can reduce detectability, provided that the initial detectability ( $\mathrm{d}^{\prime} \mathrm{sS}$ ) is reasonably good. However, although there is no indication of recognition without detection, recognition is apparently unrelated to detection performance. In other words, in

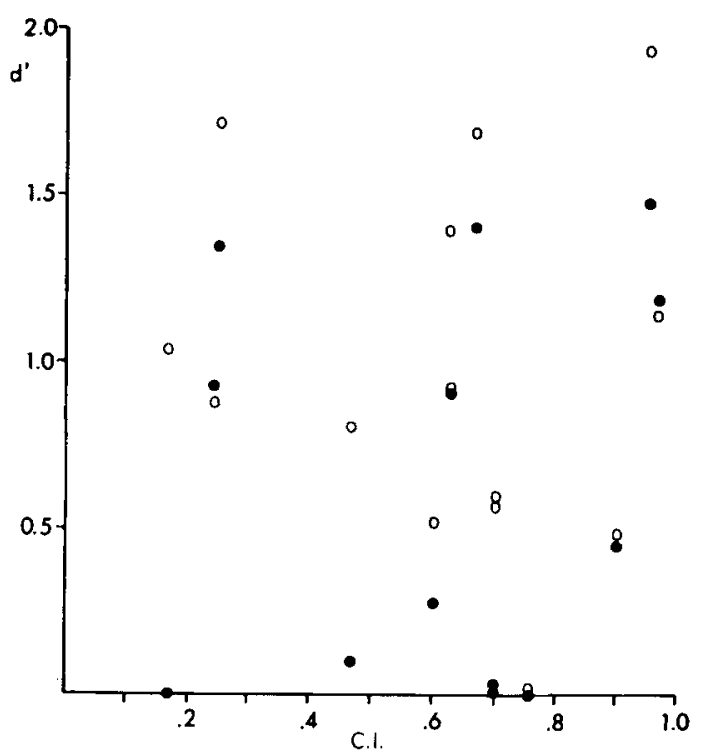

Fig. 2. $d^{\prime}$ as a function of signal confusion (CI). Open circles indicate detectability values derived from individual collapsed S-R matrices; closed circles are detectability values based on ratings.

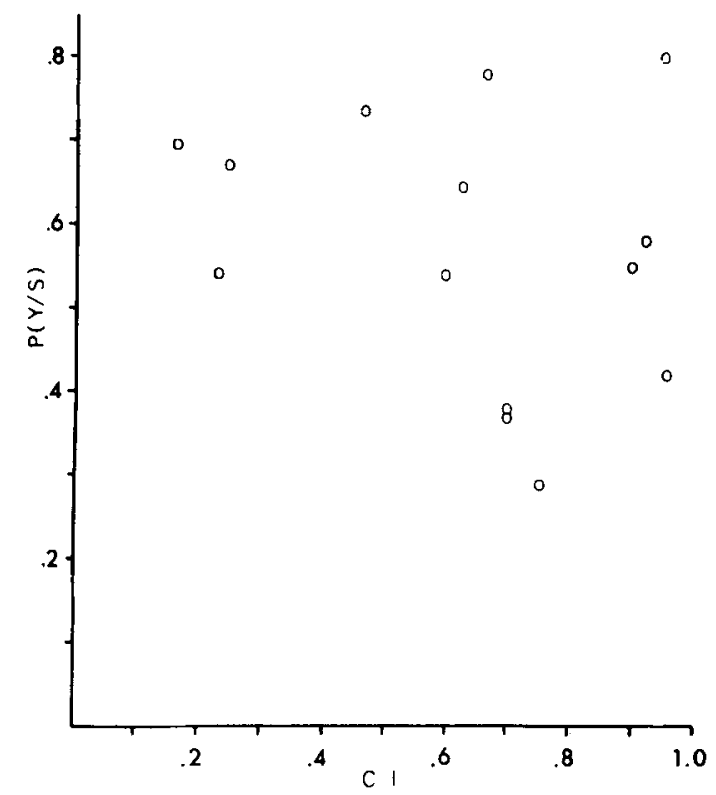

Fig. 3. $\mathrm{Cl}$ as a function of 'hit' rate, $\mathrm{p}(\mathrm{Y} / \mathrm{S})$.

this situation at least, there is little evidence for Shipley's assumption that recognition responses, either implicit or overt, are directly involved in detection performance.

\section{REFERENCES}

CREELMAN, C. D. Detection of signals of uncertain frequency. J. Acoust. Soc. Amer., 1960, 32, 775-810.

EGAN, J. P., GREENBERG, G. Z., \& SCHULMAN, A. I. Interval of time uncertainty in auditory detection. J. Acoust. Soc. Amer., 1961, 33, 771-778.

GREEN, D. M. Detection of multiple component signals in noise. J. Acoust. Soc. Amer., 1958, 30, 904-911.

GUNDY, R. F. Auditory detection of an unspecified signal. J. Acoust. Soc. Amer., 1961, 33, 1008-1012.

LUCE, R. D. Individual choice behavior. New York: Wiley, 1959.

SHIPLEY, E. F. Cueing as a determiner of apparent variability in sensitivity. J. Acoust. Soc. Amer., 1959, 31, 834.

SHIPLEY, E. F. A model for detection and recognition with signal uncertainty. Psychometrika, 1960, 25, 273-289.

SWETS, J. A. Central factors in auditory frequency selectivity. Psychol Bull, $1963,60,429-440$.

SWETS, J. A., \& BIRDSALL, T. G. The human use of information: III. Decision making in signal detection and recognition situations involving multiple alternatives. Thans. IRE Information Theory, 1956, IT-2, 138-165.

TANNER, W. P., JR., SWETS, J. A., \& GREEN, D. M. Some general properties of the hearing mechanism. University of Michigan: Electronic Defense Group, 1956, Technical Report No. 30.

VENIAR, F. A. Signal detection as a function of frequency ensemble. $J$. Acoust. Soc. Amer., 1958, 30, 1020-1024; 1075-1078.

\section{NOTE}

1. Address: Department of Psychology, University of Hull, England.

(Accepted for publication Moy 29, 1968.) 